\title{
GLOBAL TOTAL VARIATION MINIMIZATION
}

\author{
FRANÇOISE DIBOS ${ }^{1}$, GEORGES KOEPFLER ${ }^{2}$,
}

The minimization of the Total Variation is an important tool of image processing. A lot of authors have addressed the problem and developed algorithms for image denoising.

Images, seen as functions of two variables, are at most piecewise smooth and important data like edges are discontinuities. Thus image restoration techniques using regularization by smoothing will destroy on one hand noise and on the other important features (e.g. edges, textures). By working in the space of functions of bounded total variation we allow discontinuities in the result of the minimization and thus preserve sharp boundaries.

Let $u$ be a real valued function defined on an open subset $\Omega$ of $\mathbb{R}^{2}$ ( $\Omega$ is usually a rectangle). Then Rudin and Osher's BV principle consists in the following problem

$$
\text { minimize } \int_{\Omega}|\nabla u| \quad \text { subject to constraints. }
$$

The quantity we minimize, the integral of the magnitude of the gradient, will be defined precisely.

Now the minimization of the Total Variation can be achieved by a gradient descend which yields the following evolution equation:

$$
\frac{\partial u}{\partial t}=\operatorname{div}\left(\frac{\nabla u}{|\nabla u|}\right)=\operatorname{curv} u .
$$

A lot of authors have studied the $T V$ minimization problem and different approaches have been proposed. The main problem comes from the non differentiable argument $|\nabla u|$, and often regularization techniques are used.

We will present an alternative approach of the Total Variation minimization problem.

The aim of this paper is to give a sense to equation (1) for an image $u$ with bounded variation such that we can deduce an algorithm which needs no prior regularization.

To present and motivate our global minimization model of Total Variation, we reformulate equation (1) using the Coarea formula on the level sets of $u$. This leads us to consider for almost each $\lambda \in \mathbb{R}$ the following equation

$$
\int_{E_{\lambda}} \frac{\partial u}{\partial t} d x=\mathcal{P}\left(E_{\lambda}\right)
$$

where $E_{\lambda}=\{x \mid u(x)<\lambda\}$ is the level set associated to $\lambda$ and $\mathcal{P}\left(E_{\lambda}\right)$ its perimeter.

In order to be able to investigate the properties of (2) we will consider two different frameworks, in each we will be able to assure Total Variation minimization.

First we take $u \in B V(\Omega) \cap C^{\infty}(\Omega)$ and suppose that $\frac{\partial u}{\partial t}$ is constant on each level line $\partial E_{\lambda}$. We prove that (2) minimizes the Total Variation. 
Then we adapt (2) to digital image data, this leads us to an operator on digital images which diminishes the Total Variation. We give a practical algorithm and show experimental results.

1 Ceremade (URA CNRS 749), Université de Paris 9 Dauphine, Place de Lattre de Tassigny, 75775 Paris cedex 16, France

2 Prisme, UfR de Maths. et Info., Univ. de Paris 5 René Descartes, 45, Rue des Saints-Pìres, 75270 Paris Cedex 06, France 